Proceedings of the 42nd IEEE

Conference on Decision and Control

Maui, Hawaii USA, December 2003

WeA14-2

\title{
Parametric Uncertainty in Sensor Fault Detection for a Turbofan Jet Engine
}

\author{
Andreas Johansson \\ Luleå University of Technology \\ Control Engineering Group \\ SE-971 87 Luleå, Sweden \\ andreas.johansson@sm.1uth.se
}

\author{
Torbjörn Norlander \\ Volvo Aero Corporation \\ SE-46181 Trollhättan, Sweden \\ torbjorn.norlander@volvo.com
}

\begin{abstract}
A method for detecting sensor faults in a turbofan engine is presented. The proposed method consists of an observer with integral action and an adaptive detection threshold. The threshold is computed with the assumption of parametric uncertainty in the process model. Successful simulations with sensor data from an RM12 jet engine shows that the method is capable of detecting even a very small increase in sensor noise promptly without generating false alarms.
\end{abstract}

\section{INTRODUCTION}

Technical systems are inherently exposed to faults such as leaking valves, broken bearings, faulty sensors, etc. In most applications it is vital that these faults are detected and isolated in an early stage and accommodated for. In e.g. a single engine aircraft, a fault that leads to a shut down of the engine will inevitably have hazardous consequences. This is of course not allowed to happen, instead continuous operation despite any occurring faults must be ensured. As faults in mechanical systems mainly are due to wear, stress or poor quality, it is not likely that there will be less faults in the future so a good strategy for detection and handling is of great concern.

Mechanisms that prevents faults from leading to catastrophic failures are today standard when developing aircraft jet engines. The most commonly used method is to utilize hardware redundancy, typically sensor redundancy, computing redundancy and even process redundancy (more than one engine). The fault detection mechanism are in these cases based on voting or out-of-range checks but the introduction of electronic units into the control system has enabled the use of model-based fault detection methods. Model-based techniques are expected to provide improved fault detection methods, decreasing the number of aborted missions, and reduction of the need for redundant sensors, resulting in lower cost and weight, e.g. for the military low bypass turbofan engine RM12 installed in the single engine aircraft JAS-39 Gripen. See [6] for a survey a model-based fault detection applied to jet engine systems.

The idea behind model-based fault detection is to utilize the redundancy in information obtained from measurement in combination with a process model. If the measured output does not match the expected output produced by a process model, then the presence of a fault can be deduced. During the past three decades, extensive research has been carried out in this area and many methods have been developed[3]. All of these consist essentially of two steps, residual generation and residual evaluation. The purpose of the first step is to generate a signal, the residual, which is supposed to be nonzero in the presence of fault and zero otherwise. This problem has been treated extensively in the literature and solutions based on e.g. state observers, parity equations [7], or on-line identification algorithms [4] have been suggested.

However, the residual is almost always nonzero due to disturbances and model perturbations, even if there is no fault. The purpose of the second step of the fault detection algorithm is thus to evaluate the residual and draw conclusions on the presence of a fault. This is done by comparing some function of the residual to a threshold and then to declare the presence of a fault if the former exceeds the latter. The residual evaluation problem considering stochastic disturbances has been treated in e.g. [1] but the main source for nonzero residuals is often model uncertainty. In [2], robustness against unstructured, additive uncertainty in the frequency domain is achieved.

However, to be able to extend fault detection methods to uncertain, nonlinear process models, the presence of parametric uncertainty needs to be addressed. This kind of uncertainty description has not been widely considered but an exception is [5], where a system for detecting clogging in the injection lines of a pulverized coal injection plant was developed considering parametric uncertainty.

\section{A. The RM12 turbofan engine}

The turbofan RM12 (see Fig.1), which is the application in this study, is a two spool engine with a variable output nozzle, equipped with a Full Authority Digital Engine Control (FADEC) system. The RM12 FADEC is developed for a single engine application but the RM12 is also equipped with a hydromechanical backup control system for safety reasons (hardware redundancy).

The main purpose of all aircraft engines is to deliver demanded thrust. This is done in the RM12 by building up the pressure of the incoming air in two steps, with the fan (low pressure system) and the compressor (high pressure system). This compressed air is then fed from the compressor 
into the combustion chamber where the fuel is supplied and ignited. The heated gas flow is then used to drive the two turbines, high pressure turbine and low pressure turbine in respective order. The gas can be further heated after the turbines in the afterburner if needed to meet the pilots demand for thrust. At the end of the engine is a variable output nozzle which is used to adjust the operating point for the fan and also to control the low pressure outlet temperature (T5).

The control variables (see figure 1) in the RM12 are the variables guide vanes in the fan (FVG) and the compressor (CVG) respectively, the fuel flow in the burner (WFM) and the afterburner (WFR), and the variable area (A8) in the output nozzle.

In normal mode, demanded thrust is obtained by controlling the compressor speed (NH). This is achieved by computing an NH-reference from the demanded thrust (PLA - Pilot Lever Angle) and the compressor inlet temperature (T25, actually T2.5). For the PLA, there are redundant measurements, but the temperature $\mathrm{T} 25$ is measured by a single sensor only, which makes it very important to determine the health of this sensor. The current solution for RM12 is an out-of-range detection and if a sensor fault is detected then the mission is aborted and the hydromechanical backup is used to control the engine while the pilot is returning to the base.

This paper reports how analytical models can be used to improve the detection of faults in the T25-sensor. The model could also be used for redundancy purposes so that missions can be completed even with a faulty T25-sensor. The process model that is utilized is linear and time-varying but also uncertain, which motivates developing the method for dealing with parametric uncertainty that is presented in the sequel.

\section{B. Outline of the paper}

The continuation of this paper begins with some mathematical preliminaries in section II. In section III, a model for the turbofan engine and more specifically the compressor inlet temperature, T25, is described and in section IV, a residual generator with integral action is introduced for this process model. Some general remarks on the residual evaluation problem are presented in section $V$ and an adaptive threshold for evaluation of the residual of the T25 fault detection problem is calculated. The resulting fault detection method is evaluated with test-bed data from an RM12 engine in section VI while some conclusions and ideas for future work are given in section VII.

\section{PREliminaries}

When $x$ is a constant scalar, $|x|$ denotes its absolute value and when $x$ is a scalar function then $|x|$ is straightforwardly defined by $|x|(t) \triangleq|x(t)|$.

All signals, i.e. functions of time are defined on $\mathbb{R}_{+} \triangleq$ $\{t \in \mathbb{R} \mid t \geq 0\}$. Output $y$ from a causal linear system which is at rest at $t=0$ and has impulse response $g$ and input $u$ can thus be represented by the convolution integral

$$
y(t)=(g * u)(t) \triangleq \int_{0^{-}}^{t} g(t-\tau) u(\tau) d \tau
$$

Nonzero inputs to the system for $t<0$ are represented by initial conditions at $t=0^{-}$. The space of functions $u$ on $\mathbb{R}_{+}$ such that the $p$-norm

$$
\|u\|_{p} \triangleq\left(\int_{0^{-}}^{\infty}|u(\tau)|^{p} d \tau\right)^{1 / p}
$$

is bounded is denoted $\mathbb{L}_{p}$.

An inequality between two signals $f$ and $g$ is to be interpreted pointwise, so for example $f \leq g$ means $f(t) \leq$ $g(t)$ for all $t \in \mathbb{R}_{+}$.

We introduce the following class of norms, to be used extensively in the sequel.

$$
\|x\|_{w} \triangleq \sup _{t \geq 0} \int_{0^{-}}^{t} w(t-\tau)|x(\tau)| d \tau=\|w *|x|\|_{\infty}
$$

It is assumed that $w$ is nonnegative and not identically zero. Under these assumptions, it is straightforward to verify that the above is a norm.

The advantage of using this norm as disturbance bound is that, in combination with the residual evaluation operator introduced in Section V, efficient ways of calculating adaptive fault detection thresholds can be achieved as will be shown in the sequel. An elementary result that can be shown using the Hölder inequality is that $w$ may be chosen so that the space of functions $x$ such that $\|x\|_{w}<\infty$ contains the union of all $\mathbb{L}_{p}$-spaces including $\mathbb{L}_{\infty}$. This is the case if e.g. $w$ is the impulse response of a stable, strictly proper linear system or bounded in magnitude and nonzero only on a bounded interval.

Note also that the usual 1-norm and $\infty$-norm can be obtained as special cases of the above norm by $\|x\|_{1}=\|x\|_{\theta}$ and $\|x\|_{\infty}=\|x\|_{\delta}$ where $\theta$ and $\delta$ represent the Heaviside step function and the Dirac delta function, respectively.

\section{PROCESS MODEL OF THE COMPRESSOR INLET}

The temperature at the compressor inlet (T25), in the RM12, is modeled by the following linear time-varying, first order differential equation,

$$
\begin{aligned}
\dot{x}(t) & =(a(t)+\Delta) x(t)+b(t) \\
y(t) & =x(t)+e(t)
\end{aligned}
$$

where $x$ is the T25 temperature expressed in Rankine (R), $y$ is the measurement of the same temperature and $e$ is a 


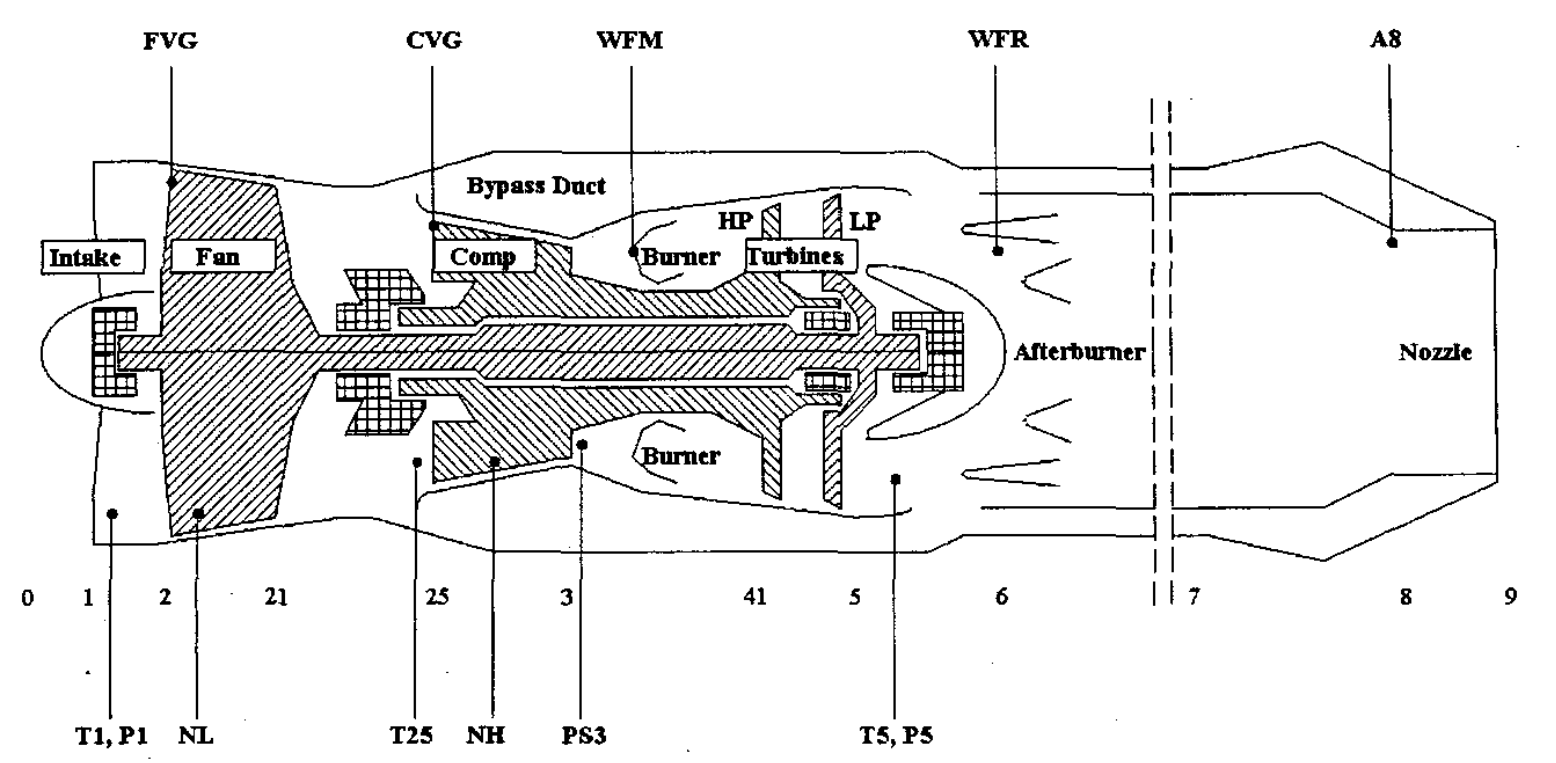

Fig. 1. Control variables (above) and measured signals (below) for the RM12. (Note also the numbering 0 to 9, this is a standard method for positioning the engine parameters)

measurement disturbance. The signals $a$ and $b$ are nonlinear functions of other measurements in the jet engine, but the details of these functions are left here. In the sequel, the time argument $t$ is dropped to enhance readability.

The uncertainty in the process model is represented by the unknown constant $\Delta$ which is assumed to be bounded by $|\Delta| \leq \delta$ for some positive $\delta$. A more realistic assumption on the uncertainty would be to allow it to vary in time in an arbitrary manner, and assuming a bound on e.g. its $\infty$ norm. This would allow $\Delta$ to make a jump when the pilot lever angle is changed drastically, which, judging from the experimental data in section VI, seems to be realistic. As will be shown later, however, the assumption on a constant uncertainty facilitates the calculations, but is still sufficient to give the desired behaviour to the adaptive detection threshold.

The measurement disturbance is assumed to be bounded in the sense of the norm (1) and the $\infty$-norm, i.e. $\|e\|_{v} \leq$ $\epsilon_{v}$ and $\|e\|_{\infty} \leq \epsilon_{\infty}$ for some weighting function $v$ and some constants $\epsilon_{v}$ and $\epsilon_{\infty}$. These assumptions on $e$ can be expressed concisely by defining the signal set $\mathbb{D}_{w}^{\epsilon}$ as the set of signals $d$ such that $\|d\|_{w} \leq \epsilon$. The assumptions on the measurement disturbance can now be formulated as

$$
e \in \mathbb{D} \triangleq \mathbb{D}_{v}^{\epsilon_{v}} \cap \mathbb{D}_{\delta}^{\epsilon_{\infty}}
$$

The task is to detect faults in the T25 sensor. Experience has shown that such faults are first manifested as increased sensor noise and the fault hypothesis is therefore defined as $\|e\|_{v}>\epsilon_{v}$.

\section{RESIDUAL GENERATION}

An observer with integral action and time-varying feedback is chosen as residual generator. The observer can be represented by

$$
\begin{aligned}
\dot{\hat{x}} & =a \hat{x}+b+\imath+K(y-\hat{y}) \\
i & =L(y-\hat{y}) \\
\hat{y} & =\hat{x}
\end{aligned}
$$

The purpose of the time-varying feedback $K(t) \triangleq a(t)+$ $k$, for some positive constant $k$, is to make the estimation error dynamics time-invariant as will be shown in the sequel. The integral action, introduced by the term $\imath$, will force the residual $r \triangleq y-\hat{y}$ to converge to zero even in the presence of the uncertainty $\Delta$. This can also be viewed as introducing high-pass filtering of the residual, which is desirable since the fault detection system is supposed to detect increased sensor noise. The observer is given the initial conditions $\hat{x}(0)=$ $y(0)$ and $\imath(0)=0$.

The dynamics of the estimation error $\tilde{x} \triangleq x-\hat{x}$ can be calculated as

$$
\begin{aligned}
\dot{\tilde{x}} & =\dot{x}-\dot{\hat{x}} \\
& =a x-\Delta x+b-a \hat{x}-b-\imath-K(y-\hat{y}) \\
& =a \tilde{x}+\Delta x-\imath-K(\tilde{x}+e) \\
& =-k \tilde{x}+\Delta x-\imath-K e
\end{aligned}
$$


Since the purpose of the integral term $\imath$ is to cancel the effects of the uncertainty $\Delta, \xi \triangleq \Delta x-\imath$ is introduced as a state variable. The dynamics of this state variable are

$$
\begin{aligned}
\dot{\xi} & =\Delta \dot{x}-\dot{i} \\
& =\Delta(a x+\Delta x+b)-L(y-\hat{y})
\end{aligned}
$$

Adding and subtracting $\imath$ in the first term of the above yields after some straightforward manipulations

$$
\dot{\xi}=\Delta(a y+b+\imath)+\Delta \xi-a \Delta e-L \tilde{x}-L e
$$

By defining the matrices

$$
\begin{gathered}
A \triangleq\left[\begin{array}{cc}
\Delta & -L \\
1 & -k
\end{array}\right] B_{1} \triangleq\left[\begin{array}{l}
1 \\
0
\end{array}\right] B_{2} \triangleq\left[\begin{array}{l}
0 \\
1
\end{array}\right] \\
C \triangleq\left[\begin{array}{ll}
0 & 1
\end{array}\right]
\end{gathered}
$$

and the state vector $z=[\xi \tilde{x}]^{T}$, the dynamics of the observer residual can be expressed as

$$
\begin{aligned}
\dot{z} & =A z-B_{1}(a \Delta+L) e-B_{2} K e+B_{1} \Delta(a y+b+\imath) \\
r & =C z+e
\end{aligned}
$$

For the calculation of the adaptive threshold in Section $\mathrm{V}$, an explicit expression for the residual is required. By defining the impulse responses $g_{1}(t)=C e^{A t} B_{1}$ and $g_{2}(t)=C e^{A t} B_{2}$, the residual $r$ can be expressed using the convolution operator as

$$
\begin{aligned}
r= & g_{1} *(\Delta(a y+b+\imath)-(a \Delta+L) e)-g_{2} *(K e)+ \\
& e+\xi(0) g_{1}+\tilde{x}(0) g_{2}
\end{aligned}
$$

with the initial values of $\xi$ and $\tilde{x}$ as

$$
\begin{aligned}
& \tilde{x}(0)=x(0)-\hat{x}(0)=y(0)-e(0)-y(0)=-e(0) \\
& \xi(0)=\Delta x(0)-\imath(0)=\Delta(y(0)-e(0))
\end{aligned}
$$

\section{RESIDUAL EVALUATION}

Due to the model uncertainties and other disturbances the residual is, in general, always nonzero. Thus, to be able to infer the existence of a fault, an evaluation signal $s$ and a threshold $\sigma$ must be defined. A fault is then declared to have occurred if $s(t)>\sigma(t)$.

A common choice of evaluation signal is the 2-norm, i.e.

$$
s(t) \equiv\|r\|_{2} \triangleq \sqrt{\int_{0}^{\infty}|r(\tau)|^{2} d \tau}
$$

The benefit of using the 2-norm is that it is then straightforward to optimize the residual generator to minimize the influence of disturbances on $s(t)$ [3].
Unfortunately, the above evaluation function can not be realized exactly, since the value of $\|r\|_{2}$ is not known until $t=\infty$ and thus $s(t)$ must therefore be modified to $e . g$.

$$
s(t)=\sqrt{\int_{0}^{t}|r(\tau)|^{2} d \tau}
$$

However, as shown in [8], the optimality of the residual generator is then lost and therefore the authors instead suggest the evaluation signal

$$
s(t) \triangleq|r(t)|
$$

In general, the evaluation signal is related to the residual by $s=\mathcal{S} r$ where the evaluation operator $\mathcal{S}$ is necessarily causal i.e. $r_{1}(\tau)=r_{2}(\tau)$ for all $\tau \leq t$ implies that $\left(\mathcal{S} r_{1}\right)(t)=$ $\left(\mathcal{S} r_{2}\right)(t)$

We propose to use the following class of evaluation operators

$$
\left(\mathcal{S}_{w} r\right)(t) \triangleq \int_{0}^{t} w(t-\tau)|r(\tau)| d \tau=w *|r|(t)
$$

The purpose of the weighting function $w$ is to increase the influence from the most recent data, e.g. by windowing or exponential forgetting.

The proposed class of evaluation operators has two major advantages; Firstly, the absolute value of the residual is likely to be less sensitive to outliers than the square and secondly, as will be shown in the sequel, it provides efficient ways of calculating the adaptive threshold $\sigma$, in particular when choosing the weighting function $w$ the same as the weighting function $v$ of the disturbance bound. Note also that the evaluation signal (4) proposed in [8] can be expressed as $s=\mathcal{S}_{\delta} r$.

\section{A. Calculation of the adaptive threshold}

To prevent false alarms caused by the measurement disturbance and the uncertainty, the threshold function $\sigma$ should satisfy

$$
\sigma(t) \geq \sup _{d \in \mathbb{D},|\Delta|<\delta}\left(\mathcal{S}_{v} r\right)(t)
$$

In order to obtain a more explicit expression for the threshold, two lemmas with properties of the convolution operator are required.

Lemma l: (a)) $|f * g| \leq|f| *|g|$

(b)) If $f(t) \geq 0$ and $h \geq g$ then $f * h \geq f * g$

Proof. The proof is straightforward using the definition of convolution and basic properties of integrals.

Lemma 2: Let $w$ be nonnegative and define

$$
\bar{u}(t) \triangleq \sup _{\tau \in[0, t]}|u(\tau)|
$$

then 
(a)) $w *|g * e| \leq\|e\|_{w}|g| * \theta$

(b)) $w *|g *(u e)| \leq\|e\|_{w}|g| * \bar{u}$

Proof. See Appendix .

An upper bound for the evaluation signal $s(t)$ can now be calculated as

$$
\begin{aligned}
s .= & \mathcal{S}_{v} r=v *|r| \\
= & v * \mid g_{1} *(\Delta(a y+b+\imath)-(a \Delta+L) e)- \\
& g_{2} *(K e)+e+\Delta(y(0)-e(0)) g_{1}-e(0) g_{2} \mid \\
\leq & v *\left|g_{1} *(\Delta(a y+b+\imath))\right|+v *\left|g_{1} *(a \Delta e)\right|+ \\
& v *\left|g_{1} *(L e)\right|+v *\left|g_{2} *(K e)\right|+v *|e|+ \\
& v *\left|\Delta y(0) g_{1}\right|+v *\left|\Delta e(0) g_{1}\right|+v *\left|e(0) g_{2}\right| \\
\leq & |\Delta| v *\left|g_{1}\right| *|a y+b+\imath|+|\Delta|\|e\|_{v}\left|g_{1}\right| * \bar{a}+ \\
& |L|\|e\|_{v}\left|g_{1}\right| * \theta+\|e\|_{v}\left|g_{2}\right| * \bar{K}+\|e\|_{v} \theta+ \\
& |\Delta||y(0)| v *\left|g_{1}\right|+|\Delta|\|e\|_{\infty} v *\left|g_{1}\right|+ \\
& \|e\|_{\infty} v *\left|g_{2}\right|
\end{aligned}
$$

where the first and the second inequality are obtained by using Lemma 1 and Lemma 2 , respectively.

The impulse responses $g_{1}$ and $g_{2}$ depend on the uncertainty $\Delta$ and are thus not completely known. Therefore, upper bounds $\gamma_{1} \geq\left|g_{1}\right|$ and $\gamma_{2} \geq\left|g_{2}\right|$ are required. Such upper bounds are, provided that $(k-\delta)^{2} / 4-L>0$,

$$
\begin{aligned}
\gamma_{1}(t) & \triangleq \frac{e^{\left(-\alpha_{2}+\beta_{2}\right) t}-e^{-\left(\alpha_{2}+\beta_{2}\right) t}}{2 \beta_{2}} \\
\gamma_{2}(t) & \triangleq \frac{\alpha_{1}-\beta_{2}}{2 \beta_{2}} e^{\left(-\alpha_{2}+\beta_{2}\right) t}+\frac{\alpha_{2}+\beta_{1}}{2 \beta_{1}} e^{-\left(\alpha_{1}+\beta_{1}\right) t}
\end{aligned}
$$

where

$$
\begin{aligned}
& \alpha_{1}=\frac{k+\delta}{2} \quad \beta_{1}=\sqrt{\frac{(k-\delta)^{2}}{4}-L} \\
& \alpha_{2}=\frac{k-\delta}{2} . \quad \beta_{2}=\sqrt{\frac{(k+\delta)^{2}}{4}-L}
\end{aligned}
$$

Showing that $\gamma_{1}$ and $\gamma_{2}$ are in fact upper bounds for $\left|g_{1}\right|$ and $\left|g_{2}\right|$ is straightforward but tedious and the proof is consequently omitted, to save space. Note, however, that convolution by $\gamma_{1}$ and $\gamma_{2}$ is straightforward to implement as filtering by linear second-order systems.

Thus by utilizing the above bounds for the impulse responses $\left|g_{1}\right|$ and $\left|g_{2}\right|$ and the bounds for the measurement disturbance $e$ and the uncertainty $\Delta$, it is found that the evaluation signal, under fault-free conditions, is bounded by

$$
\begin{aligned}
s \leq & \gamma_{1} *\left(\delta v *|a y+b+\imath|+\delta \epsilon_{v} \bar{a}+|L| \epsilon_{v} \theta\right)+\epsilon_{v} \theta+ \\
& \epsilon_{v} \gamma_{2} * \bar{K}+\delta\left(\epsilon_{\infty}+|y(0)|\right) v * \gamma_{1}+\epsilon_{\infty} v * \gamma_{2} \\
\triangleq & \sigma
\end{aligned}
$$

\section{EXPERIMENTAL RESULTS}

The fault detection algorithm described in the previous sections has been successfully tested with test-bed data from an RM12 turbofan engine. The weight of the evaluation operator was chosen to $v(t)=\mu e^{-\mu t}$. Convolution by $v$ can thus be implemented as a simple first-order transfer function, which motivates the choice. The parameters of the observer $k=5$ and $L=2$ and of the weight, $\mu=0.1$, were not optimized in any way.

The bounds of the disturbance and the uncertainty were adjusted manually so that the evaluation signal $s(t)$ does not exceed the detection threshold $\sigma(t)$ anywhere in the 2000 seconds of available test-bed data. The values are $\epsilon=0.06$, $\epsilon_{\infty}=5$, and $\delta=0.15$.

Figure 2 (top) shows the measured T25 temperature $y$ during a time interval of 130 seconds. Note the temperature jumps at time $t=65$ and time $t=100$, caused by sudden changes of the pilot lever angle. The bottom part of the same figure shows the evaluation signal $s$ (solid), calculated by (5). The corresponding detection threshold $\sigma$, calculated by (6), is represented by the dash-dotted line. Note the reaction of the evaluation signal at the time instants of the sudden changes in $\mathrm{T} 25$, due to the uncertainty in the process model. However, the detection threshold also reacts to these changes and stays above the detection signal during the whole time interval, thus creating no false alarm. The bump in the evaluation signal and the detection threshold near the beginning of the time interval is due to the initial conditions of the observer.

Figure 3 (top) shows another time interval of the measured T25 signal with simulated sensor noise added from time $t=$ 100 and onwards. Note that the noise is barely visible for the naked eye. The bottom part of the same figure shows the evaluation signal (solid) and the detection threshold (dashdotted) for the same time interval. Note that the increase in the evaluation signal due to this sensor noise causes it to exceed the detection threshold at time $t=110$ and thus the fault is detected after 10 seconds. The dotted line shows, for reference, the evaluation signal for the same data set but without noise added to the measurement signal.

\section{CONCLUSIONS AND FUTURE WORK}

A method for detecting sensor faults in a jet engine was presented. The method consists of an observer with integral action and an adaptive detection threshold, which is calculated under the assumption of parametric uncertainty in the process model.

Future developments of the method involves extending it to multivariable systems. It would then be possible to perform fault detection on several sensors and actuators simultaneously and to isolate the fault. Other issues that need to be considered are how to choose the weight function $v$ and to find numerical values for the disturbance and uncertainty bounds. 

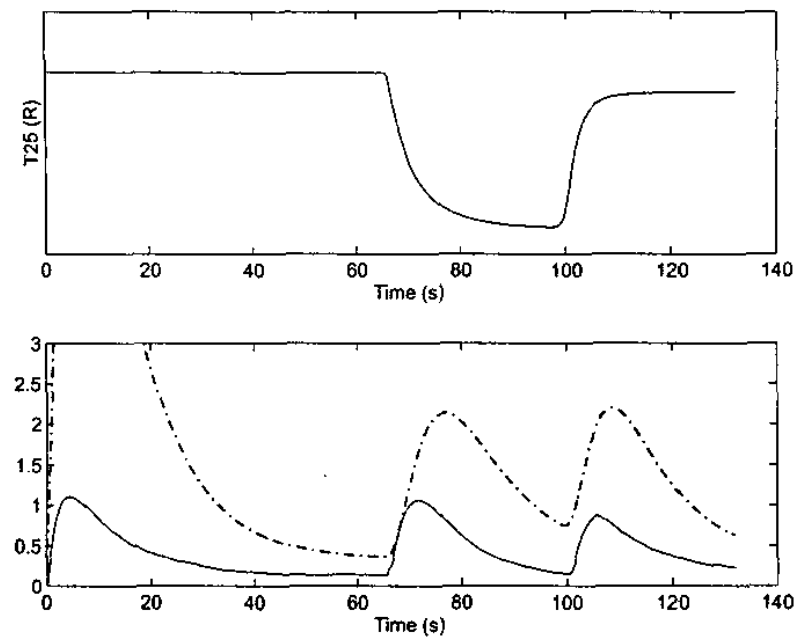

Fig. 2. Top: Measured T25 temperature. Bottom: detection threshold (dashdotted) and evaluation signal (solid)
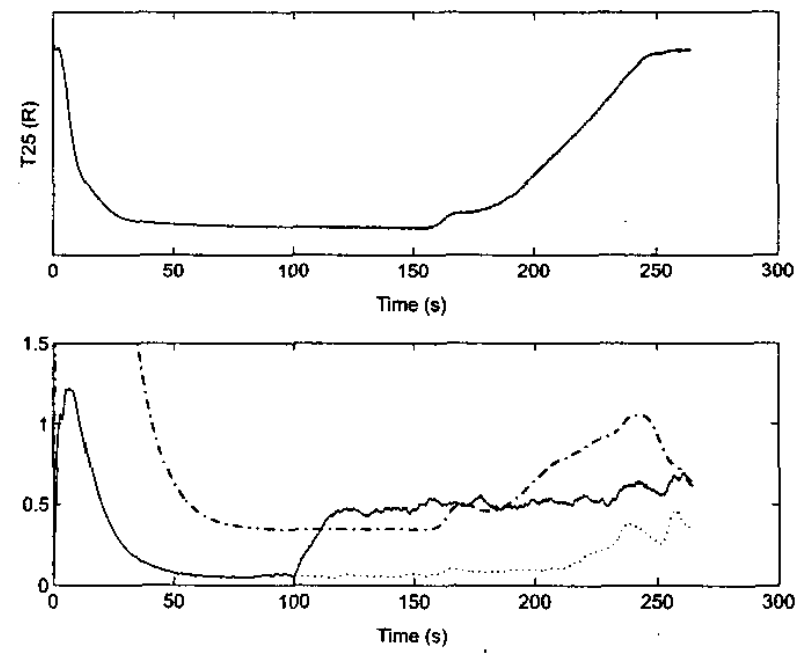

Fig. 3. Top: Measured T25 temperature with sensor fault from $t=100$. Bottom: Detection threshold (dash-dotted), evaluation signal with faul introduced at $t=100$ (solid) and without fault (dotted)

\section{ACKNOWLEDGEMENTS}

Funding of the project NFFP3-324 by the Swedish Defence Materiel Administration, FMV is gratefully acknowledged. Furthermore, the data provided by Volvo Aero Corporation is very much appreciated.

\section{REFERENCES}

[1] Michèle Basseville and Igor V. Nikiforov. Detection of Abrupt Changes. Prentice-Hall, 1993.
[2] A. Emami-Naeini, M. M. Akhter, and S. M. Rock. Effect of model uncertainty on failure detection: The threshold selector. IEEE Transactions on Automatic Control, 33(12):1106-1115, 1988.

[3] P. M. Frank and X. Ding. Survey of robust residual generation and evaluation in observer-based fault detection systems. J. Proc. Cont, 7(6):403-424, 1997.

[4] R. Isermann. Supervision, fault-detection and faultdiagnosis methods - An introduction. Control Engineering Practice, 5(5):639-652, 1997.

[5] Andreas Johansson and Alexander Medvedev. Detection of incipient clogging in pulverized coal injection lines. IEEE Transactions on Industry Applications, 36(3):877883, May/June 2000.

[6] R. J. Patton and J. Chen. Detection of faulty sensors in aero jet engine systems using robust model-based methods. In IEE Colloquium on Condition Monitoring for Fault Diagnosis, pages 2/1-2/22, 1991.

[7] N. Viswanadham, J. H. Taylor, and E. C. Luce. A frequency-domain approach to failure detection and isolation with application to GE-21 turbine engine control systems. Control - Theory and Advanced Technology, 3(1):45-72, 1987.

[8] Hao Ye, S. X. Ding, and Guizeng Wang. Integrated design of fault detection systems in time-frequency domain. IEEE Transactions on Automatic Control, 47(2):384$390,2002$.

\section{APPENDIX}

Part (b) of the lemma is proven by

$$
\begin{aligned}
(w & *|g *(u e)|)(t) \leq(|g| * w *|u e|)(t) \\
& =\int_{0}^{t}|g(t-\tau)| \int_{0}^{\tau} w(t-\theta)|u(\theta) \| e(\theta)| d \theta d \tau \\
& \leq \int_{0}^{t}|g(t-\tau)| \int_{0}^{\tau} w(t-\theta) \bar{u}(\tau)|e(\theta)| d \theta d \tau \\
& =\int_{0}^{t}|g(t-\tau)| \bar{u}(\tau) \int_{0}^{\tau} w(t-\theta)|e(\theta)| d \theta d \tau \\
& \leq \int_{0}^{t}|g(t-\tau)| \bar{u}(\tau)\|e\|_{w} d \tau \\
& =\|e\|_{w}(|g| * \bar{u})(t)
\end{aligned}
$$

where the first inequality follows from Lemma 1, the first and third equality follow from the definition of convolution. The second and third inequality follow from the definition of $\bar{u}$ and the weighted norm (1), respectively.

Part (a) of the lemma follows as a special case of part (b). 